\title{
CONCERNING POLYNOMIALS ON THE UNIT INTERVAL
}

\author{
Q. M. TARIQ
}

ABSTRACT. Let $P_{n}$ be the normed linear space of all polynomials $p$ of degree $\leq n$ such that $p(1)=0$ and $\|p\|=\left(\int_{-1}^{1}|p(x)|^{2} d x\right)^{1 / 2}$. We determine sharp upper bounds for $\left|a_{n}\right| /\|p\|$ and $\left|a_{n-1}\right| /\|p\|$ as $p(x):=\sum_{\nu=0}^{n} a_{\nu} x^{\nu}$ varies in $P_{n}$.

According to a classical result of Chebyshev if $p_{n}(x):=\sum_{\nu=0}^{n} a_{\nu} x^{\nu}$ is a polynomial of degree $n$, then

$$
\left|a_{n}\right| \leq 2^{n-1} \max _{-1 \leq x \leq 1}\left|p_{n}(x)\right|
$$

It is also known [1] that

$$
\left|a_{n}\right| \leq \frac{1 \cdot 3 \cdot 5 \cdots(2 n-1)}{n !}\left(\frac{2 n+1}{2}\right)^{1 / 2}\left(\int_{-1}^{1}\left|p_{n}(x)\right|^{2} d x\right)^{1 / 2} .
$$

In (1) equality holds for the Chebyshev polynomial $T_{n}(x):=\cos n(\arccos x)$ whereas in (2) it holds for the Legendre polynomial

$$
P_{n}(x):=\sum_{\nu=0}^{[n / 2]} \frac{(-1)^{\nu}(2 n-2 \nu) !}{2^{n} \nu !(n-\nu) !(n-2 \nu) !} x^{n-2 \nu}
$$

It was shown by Schur [2, Theorem III*] that if $p_{n}$ vanishes at one of the endpoints -1 or 1 , then (1) can be replaced by

$$
\left|a_{n}\right| \leq 2^{n-1}\left(\cos \frac{\pi}{4 n}\right)^{2 n} \max _{-1 \leq x \leq 1}\left|p_{n}(x)\right| .
$$

Here we obtain the corresponding improvement in (2). In fact, we prove

THEOREM. If $p_{n}(x):=\sum_{\nu=0}^{n} a_{\nu} x^{\nu}$ is a polynomial of degree $n$ such that $p_{n}(1)$ $=0$, then

$$
\left|a_{n}\right| \leq \frac{n}{n+1} \frac{(2 n) !}{2^{n}(n !)^{2}}\left(\frac{2 n+1}{2}\right)^{1 / 2}\left(\int_{-1}^{1}\left|p_{n}(x)\right|^{2} d x\right)^{1 / 2} .
$$

The inequality is sharp and equality holds for

$$
p_{n}(x):=P_{n}(x)-\frac{1}{n^{2}} \sum_{\nu=0}^{n-1}(2 \nu+1) P_{\nu}(x),
$$

Received by the editors October 22, 1985.

1980 Mathematics Subject Classification (1985 Revision). Primary 26C05, 26D05, 41A10, 42C15.

Key words and phrases. Polynomials on the unit interval, coefficient estimates, Chebyshev polynomials, Legendre polynomials. 
where $P_{\nu}$ is the Legendre polynomial of degree $\nu$ with the normalization $P_{\nu}(1)=1$. Besides,

(5) $\quad\left|a_{n-1}\right| \leq{\frac{\left(n^{2}+2\right)}{n+1}}^{1 / 2} \frac{(2 n-2) !}{2^{n-1}((n-1) !)^{2}}\left(\frac{2 n-1}{2}\right)^{1 / 2}\left(\int_{-1}^{1}\left|p_{n}(x)\right|^{2} d x\right)^{1 / 2}$

which is again sharp, as the following example shows:

$$
p_{n}(x):=\frac{2 n+1}{n^{2}+2} P_{n}(x)-P_{n-1}(x)+\frac{1}{n^{2}+2} \sum_{\nu=0}^{n-2}(2 \nu+1) P_{\nu}(x) .
$$

In the absence of the hypothesis $p_{n}(1)=0$ the factor $\left(n^{2}+2\right)^{1 / 2} /(n+1)$ appearing on the right-hand side of (5) is to be dropped $[1,(3)]$.

(6)

PROOF OF THE THEOREM. Let

$$
\varphi_{\nu}(x):=\left(\frac{2 \nu+1}{2}\right)^{1 / 2} P_{\nu}(x):=\left(\frac{2 \nu+1}{2}\right)^{1 / 2} \sum_{j=0}^{[\nu / 2]} \frac{(-1)^{j}(2 \nu-2 j) !}{2^{\nu} j !(\nu-j) !(\nu-2 j) !} x^{\nu-2 j}
$$

Then

$$
\int_{-1}^{1} \varphi_{\nu}(x) \varphi_{\mu}(x) d x= \begin{cases}0 & \text { if } \mu \neq \nu \\ 1 & \text { if } \mu=\nu\end{cases}
$$

and the polynomial $p_{n}(x)$ can be expressed uniquely in the form

$$
p_{n}(x)=\sum_{\nu=0}^{n} \alpha_{\nu} \varphi_{\nu}(x)
$$

where

$$
\sum_{\nu=0}^{n}\left|\alpha_{\nu}\right|^{2}=\int_{-1}^{1}\left|p_{n}(x)\right|^{2} d x
$$

From (7) in conjunction with (6) it follows that

(8)

$$
a_{n}=\left(\frac{2 n+1}{2}\right)^{1 / 2} \frac{(2 n) !}{2^{n}(n !)^{2}} \alpha_{n}, \quad a_{n-1}=\left(\frac{2 n-1}{2}\right)^{1 / 2} \frac{(2 n-2) !}{2^{n-1}((n-1) !)^{2}} \alpha_{n-1} \text {. }
$$

Now we wish to prove that if $\gamma_{\mu}>\gamma_{\nu} \geq 0$ for $\nu=0,1, \ldots, \mu-1, \mu+1, \ldots, n$ then under the hypothesis of the theorem

$$
\sum_{\nu=0}^{n} \gamma_{\nu}\left|\alpha_{\nu}\right|^{2} \leq\left(\gamma_{\mu}-\gamma\right) \sum_{\nu=0}^{n}\left|\alpha_{\nu}\right|^{2}
$$

where $\gamma$ is the unique root of the equation

$$
\sum_{\nu=0}^{n} \frac{2 \nu+1}{\gamma_{\mu}-\gamma_{\nu}-x}=0
$$

in $\left(0, \Gamma:=\min _{0 \leq \nu \leq n ; \nu \neq \mu}\left(\gamma_{\mu}-\gamma_{\nu}\right)\right)$.

We write the left-hand side of (9) as

$$
\begin{aligned}
\sum_{\nu=0}^{n} \gamma_{\nu}\left|\alpha_{\nu}\right|^{2} & =\gamma_{\mu} \sum_{\nu=0}^{n}\left|\alpha_{\nu}\right|^{2}-\sum_{\nu=0 ; \nu \neq \mu}^{n}\left(\gamma_{\mu}-\gamma_{\nu}\right)\left|\alpha_{\nu}\right|^{2} \\
& =\gamma_{\mu} \sum_{\nu=0}^{n}\left|\alpha_{\nu}\right|^{2}-\sum_{\nu=0 ; \nu \neq \mu}^{n}\left(\gamma_{\mu}-\gamma_{\nu}-\gamma\right)\left|\alpha_{\nu}\right|^{2}-\gamma \sum_{\nu=0 ; \nu \neq \mu}^{n}\left|\alpha_{\nu}\right|^{2}
\end{aligned}
$$


where, for the moment, $\gamma$ is a constant in $(0, \Gamma)$. From the hypothesis $p_{n}(1)=0$ and Schwarz' inequality we obtain

$$
\begin{aligned}
\left|\left(\frac{2 \mu+1}{2}\right)^{1 / 2} \alpha_{\mu}\right|^{2}=\left|\sum_{\nu=0 ; \nu \neq \mu}^{n}\left(\frac{2 \nu+1}{2}\right)^{1 / 2} \alpha_{\nu}\right|^{2} \leq\left\{\sum_{\nu=0 ; \nu \neq \mu}^{n}\left(\frac{2 \nu+1}{2}\right)^{1 / 2} \mid \alpha_{\nu}\right\}^{2} \\
=\left\{\sum_{\nu=0 ; \nu \neq \mu}^{n}\left(\gamma_{\mu}-\gamma_{\nu}-\gamma\right)^{1 / 2}\left|\alpha_{\nu}\right|\left(\frac{2 \nu+1}{2}\right)^{1 / 2}\left(\gamma_{\mu}-\gamma_{\nu}-\gamma\right)^{-1 / 2}\right\}^{2} \\
\leq \sum_{\nu=0 ; \nu \neq \mu}^{n}\left(\gamma_{\mu}-\gamma_{\nu}-\gamma\right)\left|\alpha_{\nu}\right|^{2} \sum_{\nu=0 ; \nu \neq \mu}^{n} \frac{2 \nu+1}{2}\left(\gamma_{\mu}-\gamma_{\nu}-\gamma\right)^{-1},
\end{aligned}
$$

so that

$$
\begin{aligned}
- & \sum_{\nu=0 ; \nu \neq \mu}^{n}\left(\gamma_{\mu}-\gamma_{\nu}-\gamma\right)\left|\alpha_{\nu}\right|^{2} \\
& \leq-\frac{2 \mu+1}{2}\left|\alpha_{\mu}\right|^{2}\left\{\sum_{\nu=0 ; \nu \neq \mu}^{n} \frac{2 \nu+1}{2}\left(\gamma_{\mu}-\gamma_{\nu}-\gamma\right)^{-1}\right\}^{-1}
\end{aligned}
$$

Now if $\gamma$ happens to be the root of the equation (10) lying in $(0, \Gamma)$, then

$$
\left\{\sum_{\nu=0 ; \nu \neq \mu}^{n} \frac{2 \nu+1}{2}\left(\gamma_{\mu}-\gamma_{\nu}-\gamma\right)^{-1}\right\}^{-1}=\frac{2}{2 \mu+1} \gamma
$$

and we get

$$
\sum_{\nu=0}^{n} \gamma_{\nu}\left|\alpha_{\nu}\right|^{2} \leq \gamma_{\mu} \sum_{\nu=0}^{n}\left|\alpha_{\mu}\right|^{2}-\gamma\left|\alpha_{\mu}\right|^{2}-\gamma \sum_{\nu=0 ; \nu \neq \mu}^{n}\left|\alpha_{\nu}\right|^{2}=\left(\gamma_{\mu}-\gamma\right) \sum_{\nu=0}^{n}\left|\alpha_{\nu}\right|^{2}
$$

which proves (9).

If $\gamma_{n}=1$ and $\gamma_{\nu}=0$ for $\nu=0,1, \ldots, n-1$, then $\gamma$ turns out to be equal to $(2 n+1) /(n+1)^{2}$ and $(9)$ reduces to

$$
\left|\alpha_{n}\right| \leq \frac{n}{n+1}\left(\sum_{\nu=0}^{n}\left|\alpha_{\nu}\right|^{2}\right)^{1 / 2}
$$

Similarly, choosing $\gamma_{n-1}=1$ and $\gamma_{\nu}=0$ for $\cdot \nu=0,1, \ldots, n-2, n$, we obtain

$$
\left|\alpha_{n-1}\right| \leq{\frac{\left(n^{2}+2\right)}{n+1}}^{1 / 2}\left(\sum_{\nu=0}^{n}\left|\alpha_{\nu}\right|^{2}\right)^{1 / 2}
$$

Combining (11), (12) with (8) we readily obtain (4), (5) respectively.

Both the inequalities (4), (5) are sharp and in each case the extremal polynomials are easily identified. 


\section{REFERENCES}

1. G. Labelle, Concerning polynomials on the unit interval, Proc. Amer. Math. Soc. 20 (1969), 321-326.

2. I. Schur, Über das Maximum des absoluten Betrages eines Polynoms in einem gegebenen Intervall, Math. Z. 4 (1919), 271-287.

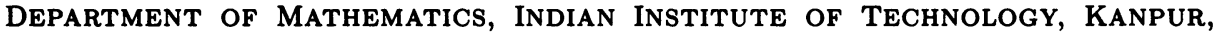
208016 U.P. INDIA 